\title{
COMPETENCIAS NUMÉRICAS TEMPRANAS DE NIÑOS ECUATORIANOS: DIFERENCIAS ENTRE TIPOS DE ESCUELAS
}

\author{
(D) Gina Bojorque ${ }^{\mathrm{I}}$ \\ (D) Joke Torbeyns II \\ (D) Jo Van Hoof III \\ (1) Daniël Van Nijlen ${ }^{\mathrm{IV}}$ \\ (D) Lieven Vershaffel ${ }^{\vee}$ \\ I Facultad de Filosofía, Universidad de Cuenca, Ecuador; gina.bojorque@ucuenca.edu.ec \\ II KU Leuven, Belgium; joke.torbeyns@kuleuven.be

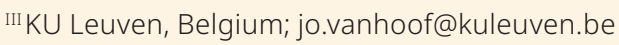 \\ Iv KU Leuven, Belgium; dvannijlen@gmail.com \\ $\checkmark$ KU Leuven, Belgium; lieven.verschaffel@kuleuven.be
}

\section{Resumen}

El objetivo de este estudio fue examinar las competencias numéricas tempranas de 355 niños ecuatorianos al iniciar el jardín infantil, teniendo en cuenta el tipo de escuela a la que asisten. Los niños resolvieron una prueba de habilidades numéricas tempranas y una de enfoque espontáneo en la numerosidad. Los resultados revelaron un nivel de habilidades numéricas tempranas y de enfoque espontáneo en la numerosidad bajos. Los niños que asisten a escuelas privadas superaron a sus compañeros de escuelas públicas rurales en habilidades numéricas tempranas, pero no en su enfoque espontáneo en la numerosidad. Se discuten estos hallazgos en vista de la importancia de prácticas de instrucción diferenciadas en matemática temprana en el jardín infantil ecuatoriano.

MATEMÁTICAS • ESCUELA ・ INFANCIA • ECUADOR

\section{COMPETÊNCIAS NUMÉRICAS PRECOCES DE CRIANÇAS EQUATORIANAS: DIFERENÇAS ENTRE TIPOS DE ESCOLAS}

\section{Resumo}

O objetivo deste estudo foi examinar as competências numéricas precoces de 355 crianças equatorianas no início do jardim de infância, considerando o tipo de escola que frequentam. As crianças resolveram um teste de habilidades numéricas precoces e um de foco espontâneo na numerosidade. Os resultados revelaram um nível baixo de habilidades numéricas precoces e de foco espontâneo na numerosidade. As crianças que frequentam escolas privadas superaram seus colegas de escolas públicas rurais em habilidades numéricas precoces, porém não no foco espontâneo na numerosidade. Esses achados são discutidos em função da importância de práticas de instrução diferenciadas em matemática precoce no jardim de infância equatoriano. 


\title{
EARLY NUMERACY SKILLS OF CHILDREN FROM ECUADOR: DIFFERENCES BETWEEN SCHOOL TYPES
}

\begin{abstract}
The aim of this study was to examine the early numerical skills of 355 Ecuadorian children at the beginning of kindergarten, considering the type of school they attend. The children solved a test of early numerical skills and one of spontaneous focus on numerosity. The results revealed a low level of early numerical skills and spontaneous focus on numerosity. Children who attend private schools outperformed their counterparts in rural public schools in their early numerical skills, but not in their spontaneous focus on numerosity. These findings are discussed based on the importance of differentiated instructional practices in early mathematics in Ecuadorian kindergartens.
\end{abstract}

MATHEMATICS $• \mathrm{SCHOOL} \cdot \mathrm{CHILDHOOD} \cdot$ ECUADOR

\section{COMPÉTENCES NUMÉRIQUES PRÉCOCES D'ENFANTS ÉQUATORIENS : DIFFÉRENCES ENTRE ÉCOLES}

\section{Résumé}

L'objectif de cette étude était d'examiner les compétences numériques précoces de 355 enfants équatoriens à leur entrée au jardin d'enfants, en fonction du type d'école. Les enfants ont passé un test de compétences numériques, ainsi qu’un test pour évaluer leurs connaissances spontanées en numératie. Les résultats ont montré un niveau faible de compétences numériques précoces et de connaissances spontanées en numératie. Les enfants des écoles privées ont dépassé ceux des écoles publiques rurales en matière de compétences numériques précoces, mais non pas en ce qui concerne les connaissances spontanées en numératie. Ces résultats sont discutés en raison de l'importance de différencier les pratiques pédagogiques en mathématiques précoces dans les jardins d'enfance équatoriens.

MATHÉMATIQUES • ÉCOLE • ENFANCE • EQUATEUR 
ARIOS ESTUDIOS COMPARATIVOS A NIVEL INTERNACIONAL (MULLIS ET AL., 2012; OECD, 2014; UNESCO, 2015) han revelado constantemente las dificultades que presentan los estudiantes sudamericanos de primaria y secundaria en el área de las matemáticas. Estas dificultades son de gran preocupación, ya que se ha demostrado que las competencias matemáticas que poseen los estudiantes de primaria y secundaria contribuyen significativamente a sus actividades profesionales posteriores (Rivera-Batiz, 1992). Estudios recientes, llevados a cabo principalmente en países europeos y norteamericanos, han demostrado el papel fundamental de las competencias numéricas tempranas de los niños pequeños en su desarrollo matemático posterior (Aunio \& Niemivirta, 2010; De Smedt et al., 2009; Duncan et al., 2007; Hannula-Sormunen, 2015; Jordan et al., 2009; Kolkman et al., 2013; Krajewski \& Schneider, 2009; Martin et al., 2014; Rathé et al., 2016). Desafortunadamente, poco se sabe sobre el desarrollo de las competencias numéricas tempranas de niños sudamericanos. Por lo expuesto, el presente estudio pretende aumentar nuestra comprensión acerca de las competencias numéricas tempranas de los niños de jardín infantil ${ }^{1}$ de Sudamérica en general y de los niños ecuatorianos en particular y, como tal, proporcionar elementos básicos para optimizar las prácticas de instrucción matemática temprana en Ecuador, y gracias a ello, mejorar el desarrollo matemático posterior de los niños ecuatorianos.

\section{Competencias numéricas tempranas}

Investigaciones previas han demostrado que, incluso antes de ingresar al jardín infantil, los niños desarrollan una gran variedad de competencias numéricas tempranas (Clements \& Sarama, 2014; Kilpatrick et al., 2001). Las competencias numéricas tempranas de los niños han sido definidas de diversas maneras (Berch, 2005); así, algunos autores se centran en las habilidades numéricas (es decir, conocimientos y habilidades numéricos; por ejemplo, habilidades para contar o para comparar cantidades), mientras que otros se enfocan en las disposiciones numéricas (por ejemplo, la inclinación de los niños a dar sentido a las situaciones numéricas, o su inclinación a centrarse espontáneamente en las características numéricas de las situaciones de la vida cotidiana), y otros se enfocan en las habilidades y disposiciones (Bojorque et al., 2017). En el resto de este artículo, se empleará el término competencias numéricas tempranas para hacer referencia tanto a las habilidades como a las disposiciones numéricas tempranas de los niños. Al emplear el término habilidadesnuméricas tempranas nos estaremos refiriendo al conocimiento y las destrezas numéricas tempranas de los niños, y con el término disposiciones numéricas tempranas nos referiremos al lado disposicional, es decir, la atención espontánea de los niños para obtener información numérica, más definida como enfoque espontáneo en la numerosidad.

Se ha demostrado que tanto las habilidades numéricas tempranas como el enfoque espontáneo en la numerosidad se desarrollan desde muy temprana edad. Así, incluso antes de cumplir los cinco años de edad, los niños son capaces, entre otras habilidades numéricas, de (1) contar verbalmente hasta el número 20 o 30; (2) contar hasta 10 objetos colocados en línea; (3) comparar, mediante el conteo, grupos de objetos (hasta cinco objetos) y determinar qué grupo es mayor; (4) sumar colecciones pequeñas de objetos (por ejemplo, tres manzanas más dos manzanas) mediante el conteo total de

1 En el presente estudio se empleará el termino "jardín infantil" para referirse a niños y niñas de cinco años de edad que asisten al nivel denominado preparatoria o primer año de básica en Ecuador. 
objetos; y (5) descomponer pequeñas cantidades de objetos (por ejemplo, saben que tres está compuesto por 1+1+1) (Baroody et al., 2006; Clements \& Sarama, 2014). Con respecto al enfoque espontáneo en la numerosidad, los estudios de Hannula y colaboradores han demostrado que los niños de tres, cuatro y cinco años de edad son capaces de enfocarse espontáneamente en el aspecto numérico de objetos a su alrededor (Hannula et al., 2005, 2010).

Durante las últimas décadas, los estudios internacionales -aunque limitados principalmente a estudios norteamericanos y europeos- han proporcionado pruebas convergentes sobre la importancia de las habilidades numéricas tempranas de los niños pequeños para su posterior éxito en matemáticas (Aunio \& Niemivirta, 2010; De Smedt et al., 2009; Duncan et al., 2007; Jordan et al., 2009; Kolkman et al., 2013; Krajewski \& Schneider, 2009; Martin et al., 2014). Según estos estudios, la comprensión acerca de la magnitud de los números, la capacidad de conteo, y los conocimientos y habilidades básicas de suma y resta, en edad preescolar y de jardín infantil, han demostrado estar relacionados de manera predictiva con el desempeño matemático durante los años de escolaridad. Desafortunadamente, también se ha demostrado que las habilidades numéricas tempranas que poseen tanto los niños de preescolar como los de jardín infantil difieren en gran medida entre niños, ya que algunos niños apenas demuestran un pequeño dominio de estas habilidades fundamentales, mientras que otros se desempeñan en el nivel más alto de dominio (Anders et al., 2012). Dichas diferencias inter-individuales en las habilidades numéricas tempranas evidentes en el preescolar y jardín infantil son preocupantes, ya que tales habilidades han demostrado ser fundamentales para el desarrollo matemático posterior de los niños.

A más de las habilidades numéricas tempranas, se ha demostrado que el enfoque espontáneo en la numerosidad de los niños de preescolar y de jardín infantil contribuye significativamente a su logro matemático posterior, incluso al término de la escuela primaria (Hannula-Sormunen, 2015; Hannula-Sormunen et al., 2015). El enfoque espontáneo en la numerosidad se refiere a un proceso espontáneo, es decir, auto-iniciado y, por lo tanto, no incitado por otros, de prestar atención a la numerosidad exacta de un conjunto de elementos en una situación no explícitamente enfocada en la matemática (Hannula et al., 2010; Hannula \& Lehtinen, 2005). Estudios longitudinales realizados en Europa, - restringidos casi exclusivamente a Finlandia - revelaron grandes diferencias interindividuales, así como estabilidad intraindividual en el enfoque espontáneo en la nu merosidad de los niños preescolares y de jardín infantil (Hannula-Sormunen, 2015; Rathé et al., 2016). Al igual que en el caso de las habilidades numéricas tempranas, las diferencias interindividuales en el enfoque espontáneo en la numerosidad observadas entre los niños en edad preescolar y de jardín infantil son preocupantes, ya que se ha demostrado que están relacionadas de manera predictiva con su desarrollo matemático futuro.

A pesar de la importancia que las habilidades numéricas tempranas y el enfoque espontáneo en la numerosidad tiene para el posterior desarrollo matemático de los niños pequeños, las competencias numéricas tempranas de los preescolares y niños de jardín infantil de Sudamérica casi no han atraído la atención de los investigadores, excepto por los estudios de Bojorque et al. $(2015,2017,2019)$, en niños ecuatorianos de 4-5 años; de Cerda et al. (2011) en niños chilenos de 5 años; de Ortiz (2009) en niños colombianos de 4-5 años; y de Paik et al. (2011) en niños peruanos de 4 años). Esto es problemático, ya que los países sudamericanos difieren en general en características socioeconómicas, políticas, educativas y culturales de los países desarrollados (United Nations, 2016) en los cuales se han realizado estudios anteriores sobre el tema. En consecuencia, la generalización de los hallazgos anteriores sobre las competencias numéricas tempranas de los niños pequeños, y especialmente su validez para los países menos desarrollados de Sudamérica, sigue siendo una pregunta abierta. Además, el contar con evidencia empírica sobre las competencias numéricas tempranas de los niños pequeños en los países de Sudamérica es muy relevante para la práctica educativa, ya que proporcionará las bases para mejorar las prácticas de instrucción matemática que permitan una estimulación matemática temprana óptima de todos los niños y, por lo tanto, que también permita un adecuado desarrollo matemático posterior. 


\section{Competencias numéricas tempranas de los niños ecuatorianos de jardín infantil}

A pesar de la limitada información, basada en investigaciones, sobre las competencias numéricas tempranas de los niños de preescolar y jardín infantil en Sudamérica, los estudios de Bojorque et al. $(2015,2017,2019)$ y Bojorque, Torbeyns, Van Nijlen et al. (2018) brindan un primer paso importante hacia la comprensión de las competencias numéricas tempranas de los niños ecuatorianos en estos niveles de educación, y proveen información acerca de posibles adaptaciones esenciales, necesarias para la instrucción matemática de los niños ecuatorianos de esta edad. En tres estudios recientes, estos autores investigaron el desarrollo de las habilidades numéricas tempranas y del enfoque espontáneo en la numerosidad en niños ecuatorianos de preescolar y de jardín infantil. Estos investigadores reportaron que existe un bajo nivel de habilidades numéricas iniciales y de enfoque espontáneo en el número de los niños ecuatorianos participantes; además, observaron un limitado desarrollo de las competencias numéricas tempranas de los niños a lo largo del jardín infantil. Más aún, estos estudios proporcionaron una evidencia tentativa acerca de la existencia de diferencias significativas entre los diferentes tipos de escuelas en relación a las competencias numéricas tempranas de los niños, favoreciendo a los niños que asisten a escuelas privadas; en otras palabras, las diferencias interindividuales en las habilidades numéricas tempranas y en el enfoque espontáneo en la numerosidad estaban presentes desde el inicio del jardín infantil y estaban asociadas con el tipo de escuela al que asistían los niños. Resultados similares fueron observados en un estudio realizado con niños brasileños de 7 a 12 años de edad, en el cual se encontró que los niños que asisten a escuelas públicas urbanas superaron a los niños de escuelas públicas rurales en tareas de procesamiento numérico ( Santos et al., 2012).

El sistema educativo ecuatoriano comprende tres niveles: (1) nivel inicial para niños de 3 a 4 años (preescolar), (2) educación básica, para niños de 5 a 14 años (el primer año de educación básica corresponde al jardín infantil), y (3) bachillerato, para estudiantes de 15 a 17 años (Ministerio de Educación, 2012). El nivel de educación básica, incluido el año de jardín infantil, está regulado por un currículo nacional obligatorio y es ofrecido principalmente por instituciones educativas públicas urbanas, públicas rurales y privadas, con aproximadamente un tercio de los niños ecuatorianos asistiendo a cada uno de estos tres tipos de escuelas (Ministerio de Educación, 2015). En general, se asume que la calidad de educación en el país es distinta entre estos diferentes tipos de escuelas, siendo las escuelas privadas las que ofrecen una educación de mayor calidad que las escuelas públicas urbanas y estas últimas las que brindan una educación de mayor calidad que las escuelas públicas rurales (PREAL, 2006). Las diferencias observadas en las competencias numéricas tempranas, ya al inicio del jardín infantil, entre los niños que asisten a escuelas públicas urbanas, públicas rurales y privadas, constituyen un reto para la instrucción matemática en el jardín infantil en Ecuador, ya que apuntan a la necesidad de una práctica de instrucción diferenciada que tenga en cuenta las necesidades educativas particulares en matemática temprana de los niños que ingresan a estos diferentes tipos de escuelas, con el fin de tratar de evitar un comienzo desigual al ingresar al primer grado (segundo de básica en el sistema educativo ecuatoriano).

\section{El presente estudio}

Teniendo en cuenta los hallazgos de Bojorque et al. (2015, 2017,2019) y Bojorque, Torbeyns, Van Nijlen et al. (2018) y sus implicaciones para comprender el desarrollo de las competencias numéricas tempranas y optimizar la instrucción matemática temprana en Ecuador, y en general en los países de Sudamérica, el primer objetivo del presente estudio fue evaluar la validez de dichos hallazgos mediante el análisis de las competencias numéricas tempranas en una muestra más amplia de niños ecuatorianos que inician el jardín 
infantil. Debido a que los estudios de Bojorque y colaboradores incluyeron solamente de cinco a diez escuelas (provenientes de los tres tipos principales de escuelas; resultando en una muestra total de alrededor de 100 niños), en el presente estudio se incluyó una muestra suficientemente grande de escuelas y niños. Esta muestra más amplia permitirá, además, realizar análisis metodológicamente válidos sobre las diferencias entre los tipos de escuela en relación a las competencias numéricas tempranas de los niños al inicio del jardín infantil y, por lo tanto, ayudará a tener una mejor comprensión de estas diferencias y sus implicaciones para la instrucción matemática temprana a través de los tipos de escuelas en el Ecuador. En consecuencia, el segundo objetivo de este estudio fue examinar si existen diferencias significativas en las competencias numéricas tempranas de los niños ecuatorianos de jardín infantil que estén asociadas al tipo de escuela a la que asisten dichos niños.

En base a los objetivos planteados, se han formulado dos grupos de preguntas de investigación. El primer grupo de preguntas se enfoca en evaluar las competencias numéricas tempranas de los niños que ingresan al jardín infantil, así como sus interrelaciones:

1a. ¿Cómo se desempeñan los niños ecuatorianos en una prueba de habilidades numéricas tempranas y de enfoque espontáneo en la numerosidad al inicio de jardín infantil?

1b. ¿Se observan relaciones entre las habilidades numéricas tempranas y el enfoque espontáneo en la numerosidad al inicio de jardín infantil?

El segundo grupo de preguntas de investigación se centra en explorar las diferencias e interrelaciones existentes en las competencias numéricas tempranas de los niños ecuatorianos al inicio del jardín infantil asociadas a los tres diferentes tipos de escuelas:

2a. ¿Los niños ecuatorianos que asisten a escuelas públicas urbanas, públicas rurales y privadas difieren en sus habilidades numéricas tempranas y su enfoque espontáneo en la numerosidad al inicio de jardín infantil?

2b. ¿Se observan diferencias en las relaciones entre las habilidades numéricas tempranas y el enfoque espontáneo en la numerosidad que estén asociadas a los tres tipos de escuelas?

Como se argumentó anteriormente, las respuestas a estas preguntas son altamente relevantes no solo en vista de una mejor comprensión teórica sobre el desarrollo de competencias numéricas tempranas en muestras de países sudamericanos, sino que también son significativas a nivel práctico, ya que proporcionan bases importantes para optimizar la instrucción matemática temprana en el jardín infantil ecuatoriano.

\section{Método}

El presente estudio es parte de un proyecto de investigación a gran escala que se centra en el desarrollo y la estimulación de las competencias numéricas tempranas de los niños ecuatorianos de jardín infantil. En vista de los objetivos del presente estudio, solamente se incluyen los instrumentos y resultados relacionados con las competencias numéricas tempranas que resultan relevantes, con especial atención a las diferencias entre tipos de escuelas relacionadas con estas competencias numéricas tempranas. En la investigación a gran escala se estudiaron, en primer lugar, las competencias numéricas (habilidades numéricas iniciales y de enfoque espontáneo en el número) de una muestra de niños ecuatorianos de jardín infantil, las cuales demostraron un bajo nivel de desarrollo (Bojorque et al., 2017; Bojorque, Torbeyns, Van Nijlen et al.2018). En vista de ello, se procedió a implementar un programa de intervención matemática temprana para estimular dichas competencias, siguiendo un diseño experimental pre testintervención-post test, con grupo de control. Después de controlar por la influencia de la memoria de trabajo la inteligencia, la edad y el nivel socioeconómico de los niños, los resultados indicaron que los niños del grupo experimental progresaron más en sus competencias numéricas tempranas que los del grupo de control, demostrándose así la efectivad de la intervención matemática (para mayor información, véase, Bojorque, Torbeyns, Van Hoof et al., 2018). 


\section{Participantes}

Los participantes fueron 355 niños ecuatorianos de jardín infantil provenientes de 18 escuelas diferentes de la región de Cuenca, provincia del Azuay. Se seleccionaron niños que inician el jardín infantil ya que el determinar el nivel desarrollo matemático en esta etapa puede ayudar a mejorar la estimulación matemática que los niños ecuatorianos reciben, con miras a optimizar su desempeño escolar futuro. Se seleccionó una muestra conveniente de escuelas, con seis escuelas por tipo de escuela (pública urbana, pública rural y privada), una clase por escuela, alrededor de 20 niños por clase. La edad media de los niños participantes fue de 5 años, 2 meses ( $\mathrm{DE}=3.7$ meses).

Sería importante explicar cómo se hace la selección de los objetos de estudio (sujetos) para validar el comienzo de la etapa de jardín de infantes y la comparación de los resultados por tipo de escuela, pero no separar los resultados por tiempo. Solo los niños que tuvieron consentimiento informado de los representantes participaron en el estudio. La Tabla 1 describe el número total de niños y su edad media por tipo de escuela.

TABLA 1

NÚMERO DE NIÑOS (HOMBRES, MUJERES) Y EDAD MEDIA POR TIPO DE ESCUELA

\begin{tabular}{lcccc}
\hline \multicolumn{1}{c}{ Tipo de escuela } & $\mathbf{N}$ & Hombres & Mujeres & Edad media $(D E)$ \\
Pública urbana & 119 & 59 & 60 & $5 \mathrm{y} 3 \mathrm{~m}(3.7 \mathrm{~m})$ \\
Pública rural & 120 & 58 & 62 & $5 \mathrm{y} 3 \mathrm{~m}(3.9 \mathrm{~m})$ \\
Privada & 116 & 62 & 54 & $5 \mathrm{y} 1 \mathrm{~m}(3.4 \mathrm{~m})$ \\
Total & 355 & 179 & 176 & $5 \mathrm{y} 2 \mathrm{~m}(3.7 \mathrm{~m})$ \\
\hline
\end{tabular}

Fuente: Elaboración de los autores.

\section{Materiales y procedimiento}

$\mathrm{Al}$ inicio del jardín infantil, todos los niños participantes fueron evaluados mediante una prueba de habilidades numéricas tempranas y una prueba de enfoque espontáneo en la numerosidad. Estos instrumentos fueron administrados individualmente en un aula tranquila en la escuela de los niños en dos días diferentes, dentro de un período de tiempo de dos semanas. La administración de la parte individual de la prueba de habilidades numéricas tempranas fue seguida por la administración de la parte colectiva de dicha prueba, la cual se realizó en el aula de niños. Para garantizar el enfoque espontáneo de los niños en la numerosidad durante la administración de la prueba respectiva, todos los niños recibieron primero la prueba de enfoque espontáneo en la numerosidad y después la prueba de habilidades numéricas tempranas.

\section{Habilidades numéricas tempranas}

Las habilidades numéricas tempranas de los niños se midieron utilizando el Test de Evaluación Numérica y Aritmética (TENA) (Bojorque et al., 2015). El TENA fue desarrollado en base al currículo ecuatoriano para el número y la aritmética de jardín infantil. El TENA consta de 54 ítems divididos en nueve subescalas (con 6 ítems por subescala), a saber: (1) cuantificadores, (2) correspondencia uno a uno, (3) relaciones de orden más que / menos que, (4) conteo, (5) identificación de la cantidad y asociación con números, (6) números ordinales, (7) lectura y escritura de numerales, (8) suma y (9) resta. El TENA consta de dos partes: una parte individual con 29 ítems que requieren principalmente una respuesta verbal (12 ítems requieren el uso de bloques pequeños para que los niños manipulen o 
para que el examinador presente la tarea), y una parte colectiva con 25 ítems que requieren el uso de papel y lápiz. Los ítems se puntúan de forma dicotómica, con una puntuación de 0 para una respuesta incorrecta y una puntuación de 1 para una respuesta correcta (puntuación máxima de la prueba $=54$ ). El Alfa de Cronbach para las puntuaciones en el TENA para la presente muestra fue de .89.

\section{Enfoque espontáneo en la numerosidad}

El enfoque espontáneo en la numerosidad se evaluó mediante la versión en español de la prueba de imitación la Pájara Elsi (Hannula \& Lehtinen, 2005). Para el efecto, se colocó una pájara de felpo en la mesa, delante del niño, junto con una caja de ocho moras de vidrio de colores colocadas al lado izquierdo de la pájara y una caja de ocho moras de vidrio de diferentes colores en el lado derecho de la pájara. El examinador comenzó la tarea señalando las cajas con moras de colores mientras decía "Esta es la pájara Elsi, y aquí hay moras [color] y acá moras [otro color]. Observa cuidadosamente lo que hago, y luego haz lo mismo que yo hice". En el primer ítem, el examinador repitió "Observa cuidadosamente lo que hago" y luego colocó dos moras rojas y una mora azul, haciendo un movimiento exagerado de la mano, en el pico de la pájara, una a la vez; las moras cayeron en el estómago de la pájara haciendo un sonido al caer. A continuación, se le indicó al niño que hiciera exactamente lo mismo que hizo el experimentador: "iListo! Ahora haz exactamente lo que yo hice". Para el segundo ítem, se usaron tres moras verdes y dos moras amarillas. El tercer y el cuarto ítem consistieron, respectivamente, en dos moras blancas y tres moras marrones, y en una mora transparente y dos moras celestes (Hannula et al., 2010).

En línea con Hannula y Lehtinen (2005), el enfoque espontáneo en la numerosidad se calificó por cada ítem en función de las respuestas de los niños (es decir, el número de moras colocadas en el pico de la pájara), así como de sus actos de cuantificación verbales y no verbales definidos como (1) pronunciación de palabras numéricas que hacen referencia al número de moras, (2) uso de los dedos para expresar el número de moras, (3) actos de conteo identificados por el susurro de palabras numéricas o por contar con los dedos, (4) otros comentarios que se refieren ya sea al número exacto de moras o a contar las mismas, (5) interpretar que el objetivo de la prueba trata de la identificación del número exacto de moras. Se calificó al niño como enfocado en la numerosidad en un ítem si éste dio la respuesta correcta (es decir, colocó en el pico de la pájara el número correcto de moras de diferente color) y / o si se observó que realizaba al menos uno de los actos de cuantificación (1-5) antes mencionados. Todos los ítems se puntuaron de forma dicotómica $(1$ o 0), lo que dio como resultado una puntuación máxima en toda la prueba de 4 puntos. El Alfa de Cronbach para las puntuaciones de la prueba de enfoque espontáneo en la numerosidad para la presente muestra fue de 0.76 .

\section{Resultados}

Los resultados serán presentados en base a los dos grupos de preguntas de investigación planteadas. En primer lugar, se describe el desempeño de los niños en las habilidades numéricas tempranas y el enfoque espontáneo en la numerosidad al inicio del jardín infantil (Pregunta de investigación 1a), así como sus interrelaciones (Pregunta de investigación 1b). A continuación, se aborda las diferencias en las habilidades numéricas tempranas y en el enfoque espontáneo en la numerosidad relacionadas con el tipo de escuela (Pregunta de investigación 2a), y diferencias por tipo de escuela en las interrelaciones existentes entre habilidades numéricas tempranas y enfoque espontáneo en la numerosidad (Pregunta de investigación $2 \mathrm{~b}$ ). Todos los análisis se realizaron a través del paquete de software estadístico SPSS, versión 23. 


\section{Desempeño en habilidades numéricas tempranas y enfoque espontáneo en la numerosidad}

Para responder a la Pregunta de investigación la, se calcularon los estadísticos descriptivos del puntaje obtenido por los niños en las pruebas de habilidades numéricas tempranas (TENA) y de enfoque espontáneo en la numerosidad (Pájara Elsi). En la Tabla 2 se presentan los desempeños de los niños en la prueba TENA y la Pájara Elsi al inicio del jardín infantil tanto de manera general como por tipo de escuela. Como se indica en la Tabla 2, los niños generalmente obtuvieron una calificación bastante baja tanto en la prueba TENA como en la prueba la Pájara Elsi, con grandes diferencias interindividuales en las puntuaciones de ambas pruebas.

En la prueba TENA, casi el 60\% de los niños (es decir, 206 de los 355 niños) respondió menos de la mitad de los ítems correctamente. Se observaron grandes diferencias interindividuales en los puntajes de TENA de los niños, que van desde un puntaje de 7 a 49. Estos puntajes bajos en general no son sorprendentes, ya que el TENA aborda los objetivos educativos para el número y la aritmética de jardín infantil que deben ser adquiridos solo al fin del año lectivo. En cuanto a la prueba de imitación la Pájara Elsi, la mayoría de los niños (es decir, 244 de los 355 niños, o el 69\%) recibió una puntuación de 0 , lo que sugiere que no se enfocaron espontáneamente en el número exacto de moras en ninguno de los ítems. Apenas el 5\% de los niños (es decir, 16 niños) recibió la puntuación máxima de 4 en esta prueba.

TABLA 2

MEDIA Y DESVIACIÓN ESTÁNDAR DE LAS PUNTUACIONES EN EL TENA Y LA PÁJARA ELSI POR TIPO DE ESCUELA

\begin{tabular}{cccc}
\hline Instrumento & Tipo de escuela & Media & DE \\
\hline & Pública urbana & 25.29 & 8.38 \\
TENA & Pública rural & 23.19 & 7.96 \\
& Privada & 27.53 & 8.96 \\
& Total & 25.32 & 8.60 \\
\hline & Pública urbana & 0.77 & 1.27 \\
Pájara Elsi & Pública rural & 0.55 & 1.04 \\
& Privada & 0.59 & 1.01 \\
& Total & 0.64 & 1.12 \\
\hline
\end{tabular}

Fuente: Elaboración de los autores.

Con relación a la Pregunta de investigación $\mathrm{lb}$, se procedió a calcular la correlación de Spearman (en vista de que los datos de la prueba la Pájara Elsi no siguieron una distribución normal). Los resultados indicaron que habilidades numéricas tempranas de los niños al inicio del jardín infantil se asociaron positivamente con su enfoque espontáneo en la numerosidad, $r_{s}(355)=0.38$, $p<.001$. Esta asociación positiva sugiere que los niños de jardín infantil con mayores habilidades numéricas tempranas tienen un mayor enfoque espontáneo en la numerosidad en comparación con sus compañeros que tienen menores habilidades numéricas tempranas.

\section{Diferencias en habilidades y disposiciones numéricas asociadas al tipo de escuela}

Teniendo en cuenta la estructura anidada de los datos de este estudio (niños anidados en las escuelas), se realizó un análisis multinivel para examinar las diferencias en las habilidades numéricas tempranas, así como en el enfoque espontáneo en la numerosidad asociada al tipo de escuela a la que asisten los niños (Pregunta de investigación 2a). Primero se analizaron las diferencias asociadas al tipo de escuela en 
los puntajes de las habilidades numéricas tempranas; estos análisis indicaron que sí existen diferencias significativas asociadas al tipo de escuela en los puntajes de los niños en el TENA, $F(2,355)=4.03$, $p=.04$ (ver Tabla 2). Más específicamente, los niños que asisten a escuelas privadas superaron a los niños que asisten a escuelas públicas rurales en el TENA al inicio del jardín infantil, $t(18.12)=2.84$, $p=.01$. Aparte de la diferencia entre escuelas privadas y escuelas públicas rurales, no se encontró ninguna otra diferencia significativa en relación al TENA, entre tipos de escuelas. En cuanto al enfoque espontáneo en la numerosidad de los niños, los análisis no revelaron diferencias significativas en los puntajes obtenidos en la prueba la Pájara Elsi entre los niños que asisten a escuelas públicas urbanas, los niños que asisten a escuelas públicas rurales y los niños que asisten a escuelas privadas, $F(2,355)=0.68$, $p=.52$ (ver Tabla 2). Los niños de los tres tipos diferentes de escuelas comenzaron el jardín infantil con un enfoque espontáneo en la numerosidad comparativamente bajo.

Finalmente, para responder a la Pregunta de investigación $2 b$, se procedió a calcular correlaciones de Spearman entre las habilidades numéricas tempranas y el enfoque espontáneo en la numerosidad por tipo de escuela. Los puntajes del TENA y la Pájara Elsi de los niños de jardín infantil se correlacionaron positivamente en cada tipo de escuela, con $r_{s}(119)=0.43, p<.01$ para escuelas públicas urbanas, $r_{s}(120)=0.41, p<.01$ para escuelas públicas rurales, $\mathrm{y} r_{s}(116)=0.31, p<.01$ para escuelas privadas. Entonces, en cada uno de los tres tipos de escuela, los niños con habilidades numéricas tempranas más altas demostraron tener un enfoque espontáneo en la numerosidad más fuerte al comienzo del jardín infantil que los niños con habilidades numéricas tempranas más bajas.

\section{Discusión}

El presente estudio tuvo como objetivo complementar los hallazgos existentes sobre las competencias numéricas tempranas de los niños pequeños, mediante el estudio de las habilidades numéricas tempranas y el enfoque espontáneo en la numerosidad de niños de jardín infantil procedentes de un país sudamericano, Ecuador, y tener en cuenta el tipo de escuela a la que asisten.

Un primer hallazgo importante del presente estudio hace referencia a las competencias numéricas tempranas débiles de los niños ecuatorianos al inicio del jardín infantil. Observamos grandes diferencias interindividuales en las habilidades numéricas tempranas y en el enfoque espontáneo en la numerosidad de los niños de 5 años, así como asociaciones positivas entre las habilidades numéricas tempranas de los niños y su enfoque espontáneo en la numerosidad, lo que confirma los resultados de estudios anteriores llevados a cabo en Ecuador (Bojorque et al., 2017; Bojorque, Torbeyns, Van Nijlen et al., 2018) y a nivel internacional (Hannula-Sormunen, 2015; Hannula et al., 2010; Jordan et al., 2009; Rathé et al., 2016). Sin embargo, los niños ecuatorianos de jardín infantil demostraron habilidades numéricas tempranas y enfoque espontáneo en la numerosidad más bajos en comparación con sus compañeros de otros países (véanse los estudios sobre habilidades numéricas tempranas de Aunio \& Niemivirta, 2010; y de enfoque espontáneo en la numerosidad de Hannula et al., 2010; Jordan et al., 2009; Rathé et al., 2016). Estos bajos niveles en las habilidades numéricas tempranas y en el enfoque espontáneo en la numerosidad son motivo de gran preocupación, ya que estudios previos realizados en países desarrollados, principalmente en Europa y Norteamérica, destacan el papel fundamental de las competencias numéricas tempranas en el desarrollo matemático posterior de los niños e incluso en sus perspectivas profesionales futuras (Aunio \& Niemivirta, 2010; De Smedt et al., 2009; Duncan et al., 2007; Hannula-Sormunen et al., 2015; Jordan et al., 2009; Kolkman et al., 2013; Krajewski \& Schneider, 2009; Martin et al., 2014; Rivera-Batiz, 1992).

En el presente estudio no se realizó un seguimiento longitudinal del desarrollo numérico de los niños de jardín infantil hasta la escuela primaria y más allá. Esto constituye una deficiencia dadas las dificultades que los estudiantes sudamericanos de primaria y secundaria demuestran en los 
estudios comparativos internacionales en el dominio de las matemáticas (Mullis et al., 2012; OECD, 2014; UNESCO, 2015). Por lo tanto, es necesario realizar estudios de seguimiento longitudinales para profundizar nuestra comprensión de la trayectoria de desarrollo numérico de estos niños de jardín infantil, desde los primeros años de la educación matemática formal hasta sus años de escuela secundaria. Más aún, se necesitan estudios longitudinales desde los años preescolares para comprender los orígenes de estas dificultades y proporcionar una imagen completa de la trayectoria de desarrollo numérico temprano de los niños ecuatorianos. Tales estudios descriptivos a largo plazo del desarrollo de competencias numéricas tempranas de los niños ecuatorianos (y más generalmente, de Sudamérica) y de los logros matemáticos posteriores, proporcionarán los conocimientos necesarios para diseñar estudios de intervención focalizados que permitan abordar las principales dificultades en el desarrollo numérico temprano y posterior. Como una vía fructífera para futuros estudios de intervención, hacemos referencia al programa de intervención TRIAD / Building Blocks de Clements y Sarama (2013; véase también, Clements \& Sarama, 2007, 2008; Sarama et al., 2008) que demostró ser eficaz para mejorar las habilidades numéricas tempranas de los niños norteamericanos de niveles socioeconómicos desfavorecidos, y que fue investigado recientemente a través de un estudio longitudinal a gran escala en jardines infantiles ecuatorianos con resultados favorables (Bojorque, Torbeyns, Van Hoof et al., 2018). Otra limitación del presente estudio es que no se obtuvo información acerca de la educación preescolar de los niños de la muestra (es decir, si asistieron o no al nivel preescolar de 3 a 4 años), lo cual pudo haber contribuido al desarrollo de las primeras habilidades numéricas. Estudios futuros deberían incluir información sobre esta importante variable.

Como segundo hallazgo importante, se observaron diferencias en las habilidades numéricas tempranas de los niños de 5 años de edad al inicio del jardín infantil relacionadas con el tipo de escuela a la que estos niños asisten, pero no se observaron dichas diferencias en su enfoque espontáneo en la numerosidad. Si bien los niños de los tres diferentes tipos de escuelas no difirieron en su (generalmente bajo) enfoque espontáneo en la información numérica en situaciones no centradas en el número, los niños que asisten a escuelas privadas obtuvieron un mejor desempeño que sus pares de escuelas públicas rurales en las habilidades numéricas tempranas al iniciar el jardín infantil.

Estos resultados sugieren que los diferentes tipos de escuelas reciben a niños con diferentes conocimientos y habilidades numéricas tempranas, lo que podría estar relacionado con diferencias en la estimulación numérica que estos niños reciben en su hogar (Lefevre et al., 2010; Lefevre et al., 2009). Si bien en este estudio no se abordó el entorno numérico del hogar de los niños participantes, teniendo en cuenta los resultados de estudios anteriores (LeFevre et al., 2010; Lefevre et al., 2009), planteamos la hipótesis de que los niños que asisten a escuelas privadas podrían haber participado en actividades numéricas tempranas más atractivas y estimulantes en el hogar en comparación a los niños de escuelas públicas rurales, lo que genera diferencias en las habilidades numéricas tempranas de estos niños al ingresar al jardín infantil (véase también, Paik et al., 2011). En vista de que no se abordó el entorno numérico del hogar de los niños de este estudio, se requieren estudios futuros para comprobar las relaciones asumidas entre el entorno numérico del hogar de los niños pequeños y el desarrollo de sus competencias numéricas tempranas, tanto en Ecuador como en los países de Sudamérica en general, y como tal, aportar a nuestra comprensión teórica de la compleja interrelación entre estas diferentes variables también en los países sudamericanos. Finalmente, estudios futuros podrían centrase en investigar las prácticas sociales, los tipos de juegos y otras situaciones de cuantificación vividas por los niños, además de la escuela, que permitan comprender y depurar aún más los resultados.

Finalmente, las diferencias en las habilidades numéricas tempranas entre los niños de escuelas privadas frente a los de escuelas públicas rurales señalan la existencia de necesidades educativas diferentes en matemáticas tempranas entre los niños que asisten a los diferentes tipos de escuelas. Estas necesidades educativas diferentes desafían las prácticas de instrucción matemática temprana actuales, 
ya que apuntan a la importancia de crear entornos de enseñanza-aprendizaje potentes para niños con diversos niveles de habilidad matemática. Teniendo en cuenta los resultados positivos de programas de intervención focalizados, como el programa de matemática temprana TRIAD / Building Blocks (Clements \& Sarama, 2013), para abordar las necesidades y dificultades diferenciadas de los niños de jardín infantil de Norteamérica - y recientemente también de Ecuador (Bojorque, Torbeyns, Van Hoof et al., 2018) - sugerimos realizar más estudios longitudinales y de intervención con el objetivo de mejorar las competencias matemáticas tempranas de los niños pequeños a través de tales programas. La estimulación temprana y oportuna de las competencias matemáticas tempranas de todos los niños, teniendo en cuenta sus necesidades educativas diferenciadas, podría ofrecer una base sólida que les permita un mejor y fluido desarrollo matemático hasta terminar los años de escuela secundaria y más adelante.

\section{Agradecimientos}

Este estudio se llevó a cabo en el Proyecto de Cooperación Científica Bilateral "Desarrollo matemático de los niños ecuatorianos de primer año de educación básica. El rol del niño y las características del entorno" auspiciado por la Secretaría de Educación Superior, Ciencia, Tecnología e Innovación (SENESCYT), Proyecto 20150008CI y Research Foundation Flanders, Proyecto G0C8715N.

\section{Referencias}

Anders, Y., Rossbach, H. G., Weinert, S., Ebert, S., Kuger, S., Lehrl, S., \& von Maurice, J. (2012). Home and preschool learning environments and their relations to the development of early numeracy skills. Early Childhood Research 2uarterly, 27(2), 231-244. https://doi.org/10.1016/j.ecresq.2011.08.003

Aunio, P., \& Niemivirta, M. (2010). Predicting children's mathematical performance in grade one by early numeracy. Learning and Individual Differences, 20(5), 427-435. https://doi.org/10.1016/j. lindif.2010.06.003

Baroody, A. J., Baroody, A. J., Lai, M., \& Mix, K. S. (2006). The development of young children's early number and operation sense and its implications for early childhood education. In B. Spodek \& O. N. Saracho (Eds.), Handbook of research on the education of young children (pp. 187-221). Routledge.

Berch, D. B. (2005). Making sense of number sense: implications for children with mathematical disabilities. Journal of Learning Disabilities, 38(4), 333-339. https://doi.org/10.1177/00222194050380040901

Bojorque, G., Torbeyns, J., Hannula-Sormunen, M., Van Nijlen, D., \& Verschaffel, L. (2017). Development of SFON in Ecuadorian Kindergartners. European Journal of Psychology of Education, 32(3), 449-462. https://doi.org/10.1007/s10212-016-0306-9

Bojorque, G., Torbeyns, J., Moscoso, J., Van Nijlen, D., \& Verschaffel, L. (2015). Early number and arithmetic performance of Ecuadorian 4-5-year-olds. Educational Studies, 565-586. https://doi.org/10.1080/030 55698.2015 .1090302

Bojorque, G., Torbeyns, J., Van Hoof, J., Van Nijlen, D., \& Verschaffel, L. (2018). Effectiveness of the Building Blocks program for enhancing Ecuadorian kindergartners' numerical competencies. Early Childhood Research 2uarterly, 44, 231-241. https://doi.org/10.1016/j.ecresq.2017.12.009

Bojorque, G., Torbeyns, J., Van Hoof, J., Van Nijlen, D., \& Verschaffel, L. (2019). Influencia del nivel socioeconómicoen eldesarrollodelascompetenciasnuméricasdelosniñosecuatorianosdejardíninfantil. Perfiles Educativos, 41(166), 90-104. https://doi.org/10.22201/iisue.24486167e.2019.166.59183

Bojorque, G., Torbeyns, J., Van Nijlen, D., \& Verschaffel, L. (2018). Ecuadorian kindergartners' numerical development: contribution of SES, quality of early mathematics education, and school type. Educação e Pesquisa, 44, 1-21. 
Cerda, G., Pérez, C., Ortega, R., Lleujo, M., \& Sanhueza, L. (2011). Fortalecimiento de competencias matemáticas tempranas en preescolares, un estudio chileno. Psychology, Society, \& Education, 3(1), 23-39. www.psye.org

Clements, D. H., \& Sarama, J. (2007). Effects of a preschool mathematics curriculum: Summative research on the Building Blocks project. Journal for Research in Mathematics Education, 38(2), 136-163. https:// doi.org/10.2307/30034954

Clements, D. H., \& Sarama, J. (2008). Experimental evaluation of the effects of a research-based preschool mathematics curriculum. American Educational Research Journal, 45(2), 443-494. https://doi. org $/ 10.3102 / 0002831207312908$

Clements, D. H., \& Sarama, J. (2013). Building Blocks-SR A, Pre-Kindergarten. McGraw-Hill.

Clements, D. H., \& Sarama, J. S. (2014). Learning and teaching early math: The learning trajectories approach (2th ed.). Routledge.

De Smedt, B., Verschaffel, L., \& Ghesquière, P. (2009). The predictive value of numerical magnitude comparison for individual differences in mathematics achievement. Journal of Experimental Child Psychology, 103(4), 469-479. https://doi.org/10.1016/j.jecp.2009.01.010

Duncan, G. J., Dowsett, C. J., Claessens, A., Magnuson, K., Huston, A. C., Klebanov, P., Pagani, L. S., Feinstein, L., Engel, M., Brooks-Gunn, J., Sexton, H., Duckworth, K., \& Japel, C. (2007). School readiness and later achievement. Developmental Psychology, 43(6), 1428-1446. https://doi.org/10.1037/00121649.43.6.1428; 10.1037/0012-1649.43.6.1428.supp (Supplemental)

Hannula-Sormunen, M. M. (2015). Spontaneous focusing on numerosity and its relation to counting and arithmetic. In R. Cohen Kados \& A. Dowker (Eds.), The Oxford handbook of numerical cognition (pp. 1689-1699). Oxford University Press. https://doi.org/10.1017/CBO9781107415324.004

Hannula, M. M., \& Lehtinen, E. (2005). Spontaneous focusing on numerosity and mathematical skills of young children.LearningandInstruction, 15(3),237-256.https://doi.org/10.1016/j.learninstruc.2005.04.005

Hannula-Sormunen, M. M., Lehtinen, E., \& Räsänen, P. (2015). Preschool children's spontaneous focusing on numerosity, subitizing, and counting skills as predictors of their mathematical performance seven years later at school. Mathematical Thinking and Learning, 17(2-3), 155-177. https://doi.org/10.1080/ 10986065.2015.1016814

Hannula, M. M., Lepola, J., \& Lehtinen, E. (2010). Spontaneous focusing on numerosity as a domain-specific predictor of arithmetical skills. Journal of Experimental Child Psychology, 107(4), 394-406. https:// doi.org/10.1016/j.jecp.2010.06.004

Hannula, M. M., Mattinen, A., \& Lehtinen, E. (2005). Does social interaction influence 3-year-old children's tendency to focus on numerosity? A quasi-experimental study in day care. In L. Verschaffel, E. De Corte, G. Kanselaar, \& M. Valcke (Eds.), Powerful learning environments for promoting deep conceptual and strategic learning (Studia Pae, pp. 63-80). Leuven University Press.

Jordan, N. C., Kaplan, D., Ramineni, C., \& Locuniak, M. N. (2009). Early math matters: Kindergarten number competence and later mathematics outcomes. Developmental Psychology, 45(3), 850-867. https://doi. org/10.1037/a0014939.

Kilpatrick, J., J., S., \& Findell, B. (2001). Adding it up. Helping children learn mathematics. Natl Academy Pr.

Kolkman, M. E., Kroesbergen, E. H., \& Leseman, P. P. M. (2013). Early numerical development and the role of non-symbolic and symbolic skills. Learning and Instruction, 25, 95-103. https://doi.org/10.1016/j. learninstruc.2012.12.001

Krajewski, K., \& Schneider, W. (2009). Early development of quantity to number-word linkage as a precursor of mathematical school achievement and mathematical difficulties: findings from a four-year longitudinal study. Learning and Instruction, 19(6), 513-526. https://doi.org/10.1016/j.learninstruc.2008.10.002

LeFevre, J.-A., Polyzoi, E., Skwarchuk, S.-L., Fast, L., \& Sowinski, C. (2010). Do home numeracy and literacy practices ofGreek and Canadian parents predict the numeracyskillsofkindergartenchildren? International Journal of Early Years Education, 18(1), 55-70. https://doi.org/10.1080/09669761003693926 
LeFevre, J. -A., Skwarchuk, S. L., Smith-Chant, B. L., Fast, L., Kamawar, D., \& Bisanz, J. (2009). Home numeracy experiences and children's math performance in the early school years. Canadian Journal of Behavioural Science, 41(2), 55-66. https://doi.org/10.1037/a0014532

Martin, R. B., Cirino, P. T., Sharp, C., \& Barnes, M. (2014). Number and counting skills in kindergarten as predictors of grade 1 mathematical skills. Learning and Individual Differences, 34, 12-23. https://doi. org/10.1016/j.lindif.2014.05.006

Ministerio de Educación. (2012). Marco Legal Educativo. Constitución de la República, Ley Orgánica de Educación Interculturaly Reglamento General. https://www.todaunavida.gob.ec/wp-content/uploads/ downloads/2013/10/ml_educativo_2012.pdf

Ministerio de Educación. (2015). Estadística educativa. Reporte de Indicadores. https://educacion.gob.ec/ estadistica-educativa/

Mullis, I., Martin, M., Foy, P., \& Arora, A. (2012). TIMSS 2011 International results in mathematics.

Organización de las Naciones Unidas para la Educación, la Ciencia y la Cultura. (2015). El laboratorio latinoamericano de evaluación de la calidad de la educación (LLECE). Los aprendizajes de los estudiantes de América Latina y el Caribe. UNESCO.

Organization for Economic Cooperation and Development. (2014). PISA 2012 results in focus: what 15-yearolds know and what they can do with what they know. OECD.

Ortiz, M. (2009). Competencia matemática en niños en edad preescolar. Psicogente, 12(22), 390-406.

Paik, J. H., van Gelderen, L., Gonzales, M., de Jong, P. F., \& Hayes, M. (2011). Cultural differences in early math skills among U.S., Taiwanese, Dutch, and Peruvian preschoolers. International Journal of Early Years Education, 19, 133-143.

Programa de Reforma Educativa en América Latina. (2006). Calidad con equidad: El desafio de la educación ecuatoriana. Informe del progreso educativo [Quality with Equity: The challenge of Ecuadorian education. Educational progress report]. PREAL.

Rathé, S., Torbeyns, J., Hannula-Sormunen, M. M., \& Verschaffel, L. (2016). Kindergartners' spontaneous focusing on numerosity in relation to their number-related utterances during numerical picture book reading. Mathematical Thinking and Learning, 18(2), 125-141. https://doi.org/10.1080/10986065.2016.1148531

Rivera-Batiz, F. (1992). Quantitative literacy and the likelihood of employment among young adults in the United States. Journal of Human Resources, 27, 313-328.

Santos, F. H., Da Silva, P. A., Ribeiro, F. S., Ribeiro Pereira Dias, A. L., Frigerio, M. C., Dellatolas, G., \& von Aster, M. (2012). Number processing and calculation in brazilian children aged 7-12 years. The Spanish Journal of Psychology, 15, 513-525.

Sarama, J., Clements, D. H., Starkey, P., Klein, A., \& Wakeley, A. (2008). Scaling up the Implementation of a Pre-Kindergarten Mathematics Curriculum: Teaching for Understanding with Trajectories and Technologies. Journal of Research on Educational Effectiveness, 1(2), 89-119. https://doi. org $/ 10.1080 / 19345740801941332$

United Nations. (2016). World economic situation and prospects 2016.

\section{Nota sobre la autoría}

Gina Bojorque: Contribuyó con la concepción y diseño del estudio; la recogida e interpretación de datos; la redacción del borrador del manuscrito; y, la aprobación final de la versión enviada para publicación; Joke Torbeyns: Contribuyó con la concepción y diseño del estudio; al interpretación de los datos recolectados; la redacción del borrador del manuscrito; y, la aprobación final de la versión enviada para publicación; Jo Van Hoof: Contribuyó con el análisis e interpretación de datos y con aportes sustanciales al contenido intelectual del borrador del manuscrito; Daniël Van Nijlen: Contribuyó con el análisis e interpretación de datos y con aportes sustanciales al contenido intelectual del borrador del manuscrito; Lieven Verschaffel: Contribuyó con la idea y diseño del estudio; la revisión crítica del contenido intelectual del borrador del manuscrito; y, la aprobación final de la versión enviada para para publicación. 


\section{Disponibilidad de datos}

Los datos não podem ser disponibilizados publicamente. La entidad que financió los estudios no permite que los datos sean colocados en un repositorio por motivos de propiedad intelectual. Por otro lado, los materiales empleados para la toma de pruebas no han sido publicados por sus autores.

Como citar este artículo

Bojorque, G., Torbeyns, J., Van Hoof, J., Van Nijlen, D., \& Vershaffel, L. (2021). Competencias numéricas tempranas de niños ecuatorianos: Diferencias entre tipos de escuelas. Cadernos de Pesquisa, 51, Artículo e06902. https://doi.org/10.1590/198053146902 